\title{
Genetic and environmental influence on asthma: a population- based study of 11,688 Danish twin pairs
}

\author{
L.R. Skadhauge*, K. Christensen**, K.O. Kyvik*, T. Sigsgaard ${ }^{+}$
}

Genetic and environmental influence on asthma: a population-based study of 11,688 Danish twin pairs. L.R. Skadhauge, K. Christensen, K.O. Kyvik, T. Sigsgaard. (C) ERS Journals Ltd 1999.

ABSTRACT: The aim of this study was to estimate the relative influence of genetic and environmental factors on the aetiology of asthma.

The classic twin study design was used to analyse data on self-reported asthma obtained by a questionnaire mailed to 34,076 individuals, aged 12-41 yrs and originating from the Danish Twin Register.

The cumulative incidence of asthma was $6.2 \%$ in the pooled sample. The data showed a decreasing cumulative incidence with age for males, but no sex or zygosity difference was observed. Substantially higher concordance rates, odds ratios and correlations for asthma were estimated in the monozygotic than in the dizygotic twins. Using biometric modelling, a model including additive genetic and nonshared environmental effects provided the best overall fit to the data. According to this model, $73 \%$ of the variation in liability to asthma was explained by genetic factors. No sex difference or age-dependency in the magnitude of genetic effects was observed.

The biometric analysis emphasized a major influence of genetic factors in the aetiology of asthma. However, a substantial part of the variation in liability to asthma is due to the impact of environmental factors specific to the individual. There is no evidence for a substantial impact of genetic dominance or the shared environment. Eur Respir J 1999; 13: 8-14.
*Dept of Genetic Epidemiology, Institute of Community Health, and **Centre for Health and Social Policy, Odense University, Odense, Denmark. ${ }^{+}$Dept of Environmental and Occupational Medicine, Aarhus University, Aarhus, Denmark.

Correspondence: L.R. Skadhauge, Dept of Genetic Epidemiology, Institute of Community Health, Odense University, Winsloewparken 15, DK-5000 Odense C, Denmark, Fax: 4565906531

Keywords: Asthma
environment
genes
twins

Received: March 111998 Accepted after revision July 181998

Supported by the Danish Asthma-Allergy Research Foundation, the Danish Health Foundation and the Danish Research Council.
Exposure to altered or increased amounts of potentially harmful environmental factors may be a contributing factor to the observed increase in the prevalence of asthma that has been reported during recent decades. A change in susceptibility due to decreased host resistance caused by an altered biological response to environmental agents may be another explanation, whereas an increased genetic susceptibility due to a change in the genetic pool is unlikely to have occurred during the short period over which the increase in prevalence has been reported.

The classic twin study comparing phenotypic similarity in monozygotic (MZ) and dizygotic (DZ) twins is a wellestablished and often used method to estimate the genetic and environmental influence on a trait. A few large-scale twin studies on asthma have been carried out in recent years. The largest study, including 13,888 Finnish twin pairs [1], was based on register linkage and the results underlined a major impact of environmental factors on the development of the disease. In contrast, questionnairebased studies have provided comparable results, emphasizing a major influence of genetic factors on the aetiology [2-4]. None of these twin studies found evidence for an impact of the shared (family) environment. However, other study designs have suggested that susceptibility to asthma may be determined early in life by environmental exposures such as passive smoking, especially maternal smoking [5], and exposure to indoor allergens [6-8], since they

For editorial comments see page 2 are frequent causes of allergy in children and associated with the development of asthma. These factors may be part of the common environment in childhood and adolescence, during which a majority of asthmatic subjects experience the onset of symptoms.

The purpose of the present study was to estimate the relative influence of genetic and environmental factors on the aetiology of asthma. Data on self-reported asthma were obtained by means of a questionnaire mailed to a population-based cohort of twins, aged 12-41 yrs at the time of the survey and originating from the nationwide Danish Twin Register. Cumulative incidences of asthma were estimated and twin similarity was assessed using concordance rates, odds ratios and correlations. Genetic and environmental variance components of liability to asthma were estimated using standard bio metric modelling.

\section{Methods}

\section{Study population}

The present study was based on the 1953-1982 birth cohorts of the Danish Twin Registry, which comprises 20,888 twin pairs, 169 triplets and four quadruplets. The method of ascertainment and characteristics of the cohort are described in detail elsewhere [9]. Zygosity was determined by questions of similarity and mistaken identity, a method that has been shown to have a misclassification rate of $<5 \%$ [10]. At the time of ascertainment, 3,270 twin 
individuals either were dead, had emigrated, had a protected address or were impossible to trace. Among 35,528 (92\%) responders to an initial questionnaire in 1991, 96\% were willing to participate in additional studies and, between March and June 1994, a questionnaire was mailed to 34,076 individuals (including 15,558 pairs). The questionnaire was answered by 29,183 twins, corresponding to an individual response rate of $86 \%$ (after one reminder). There was no difference in response rate between the birth cohorts. The responders comprised 12,352 pairs (pairwise response rate $79 \%$ ) and a total of $11,688 \mathrm{MZ}$ and $\mathrm{DZ}$ pairs $(69 \%$ of the total represented pairs) were left for the analysis of similarity and biometric modelling after the exclusion of 297 pairs of unknown zygosity and 367 pairs with incomplete or missing data on asthma. Table 1 summarizes the number of pairs of known asthma status stratified by age in two cohorts. The highest number of pairs occurred in the 1968-1982 birth cohort of MZ females (86\%) and the lowest in the 1953-1967 birth cohort of male samesex DZ twins and opposite-sex DZ twins (61\%). In the MZ and same-sex DZ pairs there were overall male:female ratios of 0.83 and 0.95 , respectively, and $\mathrm{MZ} / \mathrm{DZ}$ ratios of 0.79 and 0.90 for males and females, respectively.

\section{Questionnaire}

The seven-page questionnaire included sections on physical health, education, occupation, smoking and drinking habits. The question on asthma was prefaced with "Do you have, or have you ever had...", where asthma, hay fever and atopic dermatitis were items on a checklist. Although additional questions on asthma-like symptoms were listed, only subjects answering "yes" to asthma were considered to have or have had asthma.

\section{Analysis}

The cumulative incidence of asthma was estimated in a file constructed by randomly assigning one twin from each pair to take account of the dependency between twins. The counterpart of the file was used as a control for the estimates. To evaluate the effect of sex and age, the pairs were stratified by sex into two age groups, the 1968-1982 and 1953-1967 birth cohorts, hereafter referred to as the young and the older cohort, respectively. The Chi-squared test was used for the analysis of sex and zygosity differences within the age groups and differences between the two age categories.

Table 1. - Number of concordantly responding pairs by zygosity and cohort

\begin{tabular}{lrr}
\hline & \multicolumn{2}{c}{ Birth cohort } \\
\cline { 2 - 3 } Zygosity group & $1953-1967$ & $1968-1982$ \\
\hline MZ female & $876(76)$ & $1137(86)$ \\
DZ female & $1234(72)$ & $994(80)$ \\
MZ male & $741(67)$ & $936(83)$ \\
DZ male & $1126(61)$ & $993(76)$ \\
DZ-os & $2042(61)$ & $1609(74)$ \\
Total & $6019(66)$ & $5669(79)$ \\
\hline
\end{tabular}

Values in parenthesis are percentages of the total number of pairs represented in the group. MZ: monozygotic; DZ: dizygotic; os: opposite sex.
The similarity in MZ and DZ twins was assessed using probandwise concordance rates, odds ratios (OR) and correlations for asthma. The classic twin methodology is based on the fact that $\mathrm{MZ}$ twins have identical genotypes whereas DZ twins share, on average, half of their genes and are no more genetically related than ordinary siblings. A greater phenotypic similarity in MZ than in DZ twins is to be expected, if there is a significant genetic component in the aetiology of the disease.

The probandwise concordance rate is defined as the proportion of affected twin partner of probands. It reflects the probability of disease for one twin given that the partner twin is affected and is, thus, directly comparable to risk rates reported for other relatives [11]. The OR utilizes the additional information available from concordant non-diseased twin pairs and can be interpreted as the increased risk of asthma for one twin given the presence versus the absence of asthma in the partner twin [12].

The correlations for asthma, expressed as tetrachoric correlations due to a dichotomous outcome, were estimated under the assumption of the multifactorial threshold model [13]. This assumes that there is an underlying normally-distributed liability (susceptibility) to a disease due to genetic and environmental factors. The manifestation of a disease appears when an individual exceeds the threshold of affection on the liability distribution, and the impact of genetic and environmental effects is reflected in the similarity of the other twin's liability to disease [14]. According to standard biometric practice assuming no epistasis (genetic interlocus interaction), no gene-environment interaction or correlation and random mating, the phenotypic variance can be separated into four variance components: variance due to additive genetic effects (A), genetic dominance (D), shared (family) environment (C) and nonshared (individual-specific) environment (E) [14]. Only nonshared environment contributes to dissimilarity within MZ twin pairs because of their genetic identity,

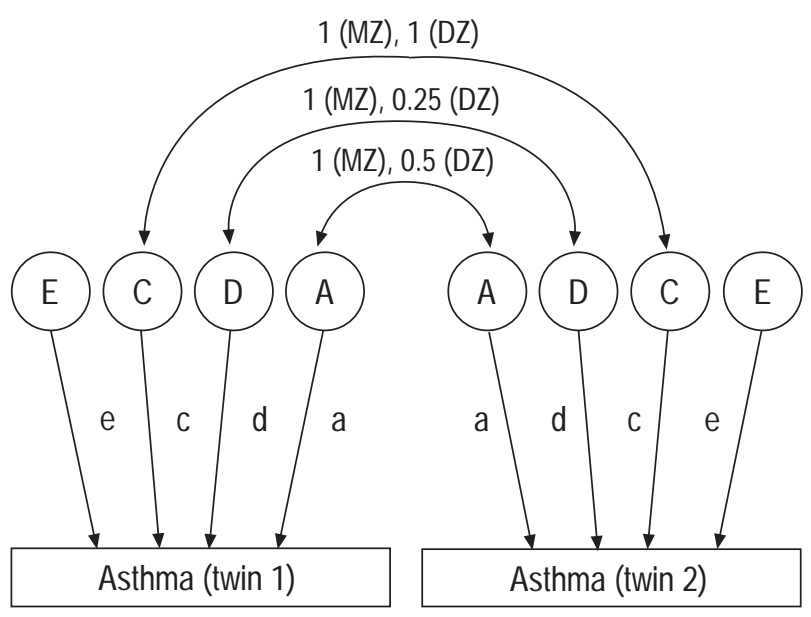

Fig. 1. - Path diagram of the causes of asthma in twins. Rectangles enclose the observed (measured) variables. Circles surround the latent (unmeasured) variables: additive genetic (A), genetic dominance (D), shared environment (C), and nonshared environment (E). Single-headed arrows define causal relationships between latent and observed variables. Double-headed arrows represent correlations between variables. Lower-case letters indicate the path coefficients (the proportion of variance in liability to asthma due to the latent variable is the square of the respective path coefficient). MZ: monozygotic; DZ: dizygotic. 
whereas the effects of additive genetic factors and genetic dominance may also contribute to dissimilarity within DZ pairs, who share, on average, half of the additive and onequarter of the dominant genetic factors (fig. 1). The tetrachoric correlations were computed from $2 \times 2$ contingency tables of disease status in twin pairs (twin A versus twin B) using the Mx software package [15].

The genetic and environmental variance components of liability to asthma and the likelihood-based confidence intervals were estimated by structural equation modelling using Mx and based on the data from the same-sex pairs only. The method has often been used with twin data and is described in detail elsewhere $[14,15]$. Because the effects of genetic dominance (D) and shared environment (C) are completely confounded in the classical study of twins reared together, it is not possible to estimate all of the parameters simultaneously in a single model. Thus, five different models, the ACE, ADE, AE, CE and E models, were fitted to the data summarized in the contingency tables. The DE model is not taken into account, since it is biologically rare to have genetic dominance in the absence of additive genetic factors. The model with the best fit is the one that fits the data well (i.e. a Chi-squared goodnessof-fit test with a nonsignificant p-value) and is the most parsimonious (i.e. none of the parameters in the model can be deleted without a significant increase in Chi-squared). For comparison of non-nested models the Akaiki Information Criterion (AIC; Chi-squared goodness-of-fit statistic minus twice the degree of freedom) was used. The model with the lowest AIC represents the best balance of goodness-of-fit and parsimony [16]. For comparison of nested models the Chi-squared difference test was used. The difference in Chi-squared of the models is itself distributed as a Chi-squared statistic with the degrees of freedom equal to the difference in the degrees of freedom of the models being compared.

Firstly, analysis of the different models, allowing the variance components to vary across sex and age, were carried out. Then, submodels of the best-fitting model, in which the variance components were constrained to be equal across sex and/or age groups, were analysed to test the effect of sex and age on the parameters. Finally, the heritability of liability to asthma (i.e. the proportion of the total phenotypic variance due to genetic variance) was derived from the best-fitting model.

\section{Results}

In total, 826 male and 950 female cases of self-reported asthma were identified, corresponding to an overall cumulative incidence of $6.2 \%(95 \%$ confidence interval (CI) $=5.81-6.59)$. The cumulative incidences of asthma in the two age categories stratified by sex and zygosity are given in table 2. There were no sex or zygosity differences in cumulative incidence within the two age groups, and no age dependency in any of the sex or zygosity groups. However, analysis across the male cohort divided into 5 yr age groups showed a statistically significant $(\mathrm{p}<0.01)$ trend in cumulative incidence for males, decreasing from $7.4 \%$ in the youngest to $5.1 \%$ in the oldest age group (data not shown). There was no trend in cumulative incidence across similar age groups for females $(p>0.2)$.
Table 2. - Cumulative incidence of asthma by sex, age and zygosity

\begin{tabular}{lccc}
\hline \multirow{2}{*}{$\begin{array}{l}\text { Age } \\
\text { yrs }\end{array}$} & $\begin{array}{c}\text { Zygosity } \\
\text { group }\end{array}$ & \multicolumn{2}{c}{ Cumulative incidence \% } \\
\cline { 3 - 4 } $12-26$ & MZ & $6.7(5.17-8.23)$ & $5.6(4.31-6.89)$ \\
& DZ-ss & $5.2(3.91-6.49)$ & $6.3(4.87-7.73)$ \\
& DZ-os & $8.6(6.77-10.42)$ & $7.0(5.40-8.60)$ \\
& UZ & $7.8(2.90-12.70)$ & $6.1(1.39-10.81)$ \\
& All & $6.7(5.83-7.57)$ & $6.3(5.49-7.11)$ \\
$27-41$ & MZ & $6.0(4.48-7.52)$ & $7.5(5.87-9.13)$ \\
& DZ-ss & $4.7(3.62-5.78)$ & $5.7(4.51-6.89)$ \\
& DZ-os & $5.8(4.49-7.11)$ & $6.8(5.52-8.01)$ \\
& UZ & $5.9(1.33-10.47)$ & $10.1(3.46-16.75)$ \\
& All & $5.4(4.68-6.12)$ & $6.6(5.87-7.33)$ \\
$12-41$ & All & $6.0(5.44-6.56)$ & $6.5(5.94-7.06)$ \\
\hline
\end{tabular}

Ninety-five per cent confidence intervals (CIs) are shown in parenthesis. The cumulative incidences and 95\% CIs were calculated using one twin from each pair. MZ: monozygotic; DZ: dizygotic; ss: same-sex; os: opposite sex; UZ: unknown zygosity.

The probandwise concordance rates, OR and tetrachoric correlations are given in table 3 . The concordance rates for asthma were significantly higher for both male and female MZ twins than for same-sex DZ twins, according to the CI, suggesting a genetic influence on the aetiology of asthma in both sexes. The highest rate was in the older cohort of male $\mathrm{MZ}$ twins, with a probability of 0.51 for one twin having asthma given the partner twin was affected. The lowest rate was 0.07 in the older cohort of opposite-sex DZ twins. No effect of sex or age was observed in the two cohorts, except for female DZ twins, where there was a significantly higher concordance in the younger cohort. The OR for asthma was also significantly higher for $\mathrm{MZ}$ than for $\mathrm{DZ}$ twins. The highest OR was among the twin partners of asthmatic male MZ twins in the older cohort, who had an OR of 37.2 for having asthma compared with the twin partners of nonasthmatic twins. By comparison, the OR was 5.1 in the group of older male DZ twins. The lowest OR was 1.3, in the older cohort of opposite-sex DZ twins. There was no effect of sex or age in the two cohorts. As expected, the tetrachoric correlations showed the same sex and age pattern as the probandwise concordance rates with regard to the differences between MZ and DZ twins.

The outcome of the biometric modelling allowing the variance components to vary across sex and age in the five models is given with the test statistics in table 4. Models that included only environmental factors (CE and $\mathrm{E}$ models) provided a very poor fit to the data $(\mathrm{p}<0.001)$. Among the models including both genetic and environmental effects, which all fitted the data well $(p>0.1)$, a model including additive genetic and nonshared environment effects (AE model) provided the best overall fit according to the AIC. The result of a Chi-squared difference test between this model and the ACE model, which includes the impact of shared environment, was nonsignificant ( $p>0.2$ ) and $C$ could therefore be excluded. The impact of dominant genetic effects could be excluded from the ADE model in a similar way, without a significant increase in Chi-squared. 
Table 3. - Probandwise concordance rate, odds ratio (OR) and tetrachoric correlation for asthma by zygosity group and age

\begin{tabular}{llcccrc}
\hline $\begin{array}{l}\text { Age } \\
\text { yrs }\end{array}$ & $\begin{array}{c}\text { Zygosity } \\
\text { group }\end{array}$ & $\begin{array}{c}\text { Concordant } \\
\text { pairs }\end{array}$ & $\begin{array}{c}\text { Discordant } \\
\text { pairs }\end{array}$ & $\begin{array}{c}\text { Probandwise } \\
\text { concordance* }\end{array}$ & OR $^{\dagger}$ & $\begin{array}{c}\text { Tetrachoric } \\
\text { correlation }\end{array}$ \\
\hline $12-26$ & MZ-F & 29 & 81 & $0.42(0.34-0.50)$ & $18.2(11.7-28.4)$ & $0.71 \pm 0.06$ \\
& DZ-F & 18 & 103 & $0.26(0.18-0.34)$ & $6.0(3.5-10.4)$ & $0.47 \pm 0.08$ \\
& MZ-M & 29 & 63 & $0.48(0.40-0.58)$ & $24.8(15.6-39.4)$ & $0.76 \pm 0.05$ \\
& DZ-M & 12 & 101 & $0.19(0.11-0.27)$ & $4.1(2.2-7.8)$ & $0.36 \pm 0.09$ \\
& DZ-os & 18 & 194 & $0.16(0.08-0.22)$ & $2.7(1.6-4.6)$ & $0.25 \pm 0.08$ \\
$27-41$ & MZ-F & 21 & 70 & $0.38(0.28-0.48)$ & $13.7(8.2-23.0)$ & $0.65 \pm 0.07$ \\
& DZ-F & 6 & 118 & $0.09(0.03-0.15)$ & $2.0(0.8-4.9)$ & $0.15 \pm 0.11$ \\
& MZ-M & 21 & 40 & $0.51(0.39-0.63)$ & $37.2(21.8-63.5)$ & $0.81 \pm 0.05$ \\
& DZ-M & 8 & 82 & $0.16(0.08-0.24)$ & $5.1(2.4-10.7)$ & $0.37 \pm 0.10$ \\
& DZ-os & 8 & 217 & $0.07(0.03-0.11)$ & $1.3(0.6-2.8)$ & $0.05 \pm 0.09$ \\
\hline
\end{tabular}

Values in parenthesis are $95 \%$ confidence intervals. Tetrachoric correlation value are mean \pm SE. *: based on SE for one proportion; ${ }^{\dagger}$ : testbased (OR ${ }^{1 \pm 1.96 / x}$, where $x$ is the square root of the Chi-squared test). MZ: monozygotic; DZ: dizygotic; F: female; M: male; os: opposite sex.

The test statistics of the AE submodels, with the variance components constrained for sex and/or age, are given in table 5. Among these models, which all provided a good fit to the data, a model in which sex and age were fixed provided the best fit to the data compared with the full AE model (Chi-squared difference $=5.3,3$ degrees of freedom (df), $p>0.1$ ). From this model, a heritability of 0.73 was derived. The confidence interval was $0.32-0.78$ based on the ADE model $\left(\mathrm{d}^{2}=0,95 \% \mathrm{CI}=0.00-0.41\right.$; $\mathrm{e}^{2}=0.27,95 \%$ CI $=0.22-0.33$ ) and $0.52-0.78$ based on the ACE model $\left(\mathrm{c}^{2}=0,95 \%\right.$ CI $=0.00-0.18 ; \mathrm{e}^{2}=0.27,95 \%$ $\mathrm{CI}=0.22-0.33$ ). Ignoring the genetic dominance or common environment completely will result in an unrealistically small CI for the heritability estimate.

\section{Discussion}

By means of a questionnaire administered to 34,076 individuals, aged 12-41 yrs and originating from the nationwide Danish Twin Register, a cumulative incidence of $6.2 \%$ was estimated for self-reported asthma. Although no overall sex difference was seen, there was a decreasing cumulative incidence with age for males but no trend across the cohort among females. Analysis for similarity between 11,688 concordantly responding twin pairs showed substantially higher concordance rates, OR, and correlations for asthma in MZ than in DZ twins, emphasizing a genetic influence on the aetiology of the disease. Biometric modelling provided evidence for a major impact of additive

Table 4. - Multigroup analysis of biometrical models for asthma: variance components and model fit statistics

\begin{tabular}{|c|c|c|c|c|c|c|}
\hline \multirow[b]{2}{*}{ Group } & \multirow[b]{2}{*}{ Variance component } & \multicolumn{5}{|c|}{ Model } \\
\hline & & $\mathrm{ACE}$ & $\mathrm{ADE}$ & $\mathrm{AE}^{*}$ & $\mathrm{CE}$ & $\mathrm{E}$ \\
\hline \multirow[t]{4}{*}{ F $12-26$ yrs } & $a^{2}$ & $0.50(0.13-0.80)$ & $0.73(0.34-0.82)$ & $0.73(0.62-0.82)$ & - & - \\
\hline & $d^{2}$ & - & $0.00(0.00-0.40)$ & - & - & - \\
\hline & $c^{2}$ & $0.21(0.00-0.52)$ & - & - & $0.60(0.50-0.69)$ & - \\
\hline & $\mathrm{e}^{2}$ & $0.29(0.19-0.41)$ & $0.27(0.18-0.38)$ & $0.27(0.18-0.38)$ & $0.40(0.31-0.50)$ & 1.00 \\
\hline \multirow{4}{*}{ F $27-41 \mathrm{yrs}$} & $a^{2}$ & $0.61(0.34-0.73)$ & $0.00(0.00-0.69)$ & $0.61(0.46-0.73)$ & - & - \\
\hline & $d^{2}$ & - & $0.64(0.49-0.76)$ & - & - & - \\
\hline & $c^{2}$ & $0.00(0.00-0.21)$ & - & - & $0.44(0.31-0.56)$ & - \\
\hline & $e^{2}$ & $0.39(0.27-0.54)$ & $0.36(0.24-0.51)$ & $0.39(0.27-0.54)$ & $0.56(0.44-0.69)$ & 1.00 \\
\hline \multirow[t]{4}{*}{ M 12-26 yrs } & $a^{2}$ & $0.76(0.40-0.85)$ & $0.69(0.00-0.84)$ & $0.76(0.65-0.85)$ & - & - \\
\hline & $d^{2}$ & - & $0.07(0.00-0.81)$ & - & - & - \\
\hline & $c^{2}$ & $0.00(0.00-0.32)$ & - & - & $0.60(0.50-0.69)$ & - \\
\hline & $\mathrm{e}^{2}$ & $0.24(0.15-0.35)$ & $0.24(0.15-0.35)$ & $0.24(0.15-0.35)$ & $0.40(0.31-0.51)$ & 1.00 \\
\hline \multirow{4}{*}{ M 27-41 yrs } & $a^{2}$ & $0.79(0.37-0.88)$ & $0.74(0.00-0.88)$ & $0.79(0.67-0.88)$ & - & - \\
\hline & $d^{2}$ & - & $0.06(0.00-0.86)$ & - & - & - \\
\hline & $c^{2}$ & $0.00(0.00-0.37)$ & - & - & $0.62(0.50-0.72)$ & - \\
\hline & $\mathrm{e}^{2}$ & $0.21(0.11-0.33)$ & $0.20(0.11-0.33)$ & $0.21(0.12-0.33)$ & $0.38(0.27-0.50)$ & 1.00 \\
\hline \multirow[t]{4}{*}{ Test statistic } & $\chi^{2}$ & 17.4 & 15.4 & 18.8 & 64.9 & 15,480 \\
\hline & $\mathrm{df}$ & 12 & 12 & 16 & 16 & 24 \\
\hline & $\mathrm{p}$ & 0.14 & 0.22 & 0.28 & $<0.001$ & $<0.001$ \\
\hline & AIC & -6.6 & -8.6 & -13.2 & 32.9 & 15,432 \\
\hline
\end{tabular}

Values in parenthesis are likelihood-based $95 \%$ confidence intervals. The variables are additive genetic (A); genetic dominance $(\mathrm{D})$; shared environment $(\mathrm{C})$; and nonshared environment $(\mathrm{E})$. The proportions of variance in liability to asthma due to $\mathrm{A}, \mathrm{D}, \mathrm{C}$ and $\mathrm{E}$ are $\mathrm{a}^{2}$, $\mathrm{d}^{2}, \mathrm{c}^{2}$, and $\mathrm{e}^{2}$, respectively. F: female; M: male; df: degrees of freedom; AIC: Akaiki Information Criterion $\left(=\chi^{2}-2 \mathrm{df}\right) .{ }^{*}$ : best fitting model by AIC. 
Table 5. - Model fit statistics for the AE model with genetic and environmental variance components constrained to be equal across sex and/or age

\begin{tabular}{lccc}
\hline Test statistics & Sex fixed & Age fixed* & Sex andage \\
\hline$c^{2}$ & 23.5 & 21.2 & 24.1 \\
df & 18 & 18 & 19 \\
p-value & 0.17 & 0.27 & 0.19 \\
AIC & -12.5 & -14.8 & -13.9 \\
\hline
\end{tabular}

A: additive genetic; E: nonshared environment; df: degrees of freedom; AIC: Akaiki Information Criterion. *: best fitting model by AIC.

genetic effects on liability to asthma, but a substantial part of the phenotypic variance was due to nonshared environmental effects. Although an impact of shared environment was seen in the young half of the female cohort, it was not statistically significant, suggesting that the familial aggregation of asthma is primarily of genetic origin. No substantial sex- or age-dependency in the magnitude of genetic effects was observed.

A few other large-scale twin studies on asthma have been carried out, primarily in the Nordic countries. In 1971 EDFORS LuBS [17] published a study of allergy among 7,000 pairs of Swedish twins, aged 42-81 yrs at the time of the questionnaire. The cumulative incidence of asthma was $3.8 \%$ and the pairwise concordance rates were 0.19 and 0.048 for MZ and DZ twins, respectively. No age trend was observed, possibly due to recall bias, as was noted in the paper. A very low cumulative incidence $(1.9 \%)$ and low probandwise concordance rates of 0.13 for $\mathrm{MZ}$ and 0.065 for DZ twins were also found in a 10-yr follow-up study among 13,888 Finnish twin pairs, aged 18-70 yrs and older at the beginning of the follow-up [1]. Cases of asthma were obtained by linkage of death certificates, hospital and pharmacy records; thus, only adult and the most severe cases were identified. Cumulative incidence, concordance rates and correlations consistent with the estimates in the present study were found in a newly published study on asthma among 2,559 Norwegian twin pairs, aged 18-25 yrs [2]. Despite a widening of the phenotype and a cumulative incidence of asthma/wheezing of $13.2 \%$, comparable estimates of correlation rates were also found in a study of 3,808 twin pairs, aged 18-88 yrs from the Australian National Health and Medical Research Council Twin Registry of volunteers [4] and in a group of 1,496 adolescent twin pairs, aged 13-20 yrs from the Dutch Twin Register, where $11.3 \%$ claimed to suffer or have suffered from asthma or bronchitis [3]. None of these studies have reported evidence for an impact of shared environment on the aetiology of asthma, although a majority of asthmatic cases experience the onset of symptoms in childhood or adolescence, when potential risk factors for asthma (e.g. mites, pets and parental smoking) may be part of the shared environment. In the Norwegian twin study, more than $80 \%$ of the asthmatic cases had occurred by 15 yrs of age, when $93 \%$ of the respondents still lived at home [2]. In the present study, an effect of the shared environment was only shown in the young female cohort, accounting for $21 \%$ of the total variation in the ACE model. However, it is unlikely that only this group, and not the corresponding group of young males, shows a shared environment effect to this extent. In addition, if the shared environment is an important factor for the development of asthma, the concordance rates, OR and correlations in MZ and DZ twin pairs would be expected to be more similar than were the case, assuming no difference in the exposures to risk factors for asthma in the two zygosity groups. However, concern has been raised about the limited power of the twin method to distinguish between shared environmental factors and additive genetic factors. This is indicated by the large CIs around C. Comparison of the ACE model with the $\mathrm{CE}$ and $\mathrm{AE}$ models has been recommended to estimate and test the significance of A versus $C[18]$. The $\mathrm{CE}$ model in the present study provided a very poor fit to the data, in contrast to the ACE model, which was not significantly different from the best-fitting AE model.

The 1953-1982 birth cohort of the Danish Twin Register is population based and has a very high degree of ascertainment [9], thus minimizing the risk of selection bias that may be present in studies based on volunteers. The young cohort is nearly complete (97\%) and since the twins were ascertained by means of the Danish Civil Registration System without knowledge of zygosity or disease status, the older cohort of twins is representative despite a lower degree of ascertainment. As the twins are registered at birth one can also ignore "the healthy twin effect" that may arise in twin registers truncated to include only pairs where both twins are still alive at a certain age.

Selection bias may arise and influence the concordance rates if the prevalence of asthma among the responders differs from the nonresponders, especially if mortality is associated with asthma. A 17-yr follow-up study of 13,540 individuals, aged $\geq 20 \mathrm{yrs}$, randomly selected from the population of the city of Copenhagen [19], showed that selfreported asthma was associated with a slight increase in mortality. Investigation of a sample of nonresponders, in this study, revealed a slightly higher prevalence of asthma than in responders. A follow-up study among 31,110 Finnish adults, mostly twins, showed a worse survival in adults with physician-diagnosed asthma than in those without, although male asthmatic subjects under 40 yrs of age did not have increased mortality [20]. Despite a high individual response rate $(86 \%)$ in the present study, only $69 \%$ of the total represented pairs were left for the analysis of correlation and biometric modelling after the exclusion of twins of unknown zygosity, twins with missing data on asthma and discordantly participating twins. In particular, male DZ and opposite-sex DZ twins from the older cohort had a low representation of intact pairs. However, no difference in cumulative incidence was found between individuals from the groups of intact pairs and the pooled sample, suggesting that selection bias with regard to asthma is negligible.

Misclassification may be another source of bias potentially introduced with the determination of zygosity and disease. Determination of zygosity by means of questionnaire is a reliable method, with a misclassification rate of $<5 \%$ by comparison between questionnaire and blood group analysis [10]. Since the zygosity has been determined previously and is therefore independent of disease status, no marked bias is introduced with regard to asthma. More obvious are the difficulties involved in the estimation of the "true" asthmatic subjects. Epidemiological as well as clinical research on asthma is handicapped by the lack of a generally accepted "gold standard" for the definition of asthma. Self-reported methodology may be biased 
by poor questionnaires and poor or different perception of the questions depending on age, sex, geographical area, social class or simply bad timing of the questionnaire (especially for seasonal asthma). In addition, secular trends may be biased owing to changes in diagnostic criteria [21, $22]$ or increased awareness of asthma over time. The outcome of the present study was solely based on the individuals' perception of having or ever having had asthma and the cumulative incidence of true asthmatic subjects may be overestimated in this population. Although the question "Have you ever had asthma?" seems to be one of the most reliable questions in epidemiological research on asthma when a questionnaire is the only diagnostic tool [23], there may be a poor correlation between the diagnosis of asthma based on clinical evaluation by a physician and that obtained by a questionnaire. Misclassification (e.g. measurement or diagnostic error) is included in the non-shared environmental effects [14] and will, therefore, underestimate the magnitude of genetic effects if present.

One of the assumptions of the classic twin study design is that the cumulative incidence of the trait under study is equal in $\mathrm{MZ}$ and $\mathrm{DZ}$ twins. No difference in cumulative incidence was found in any of the groups being compared in the present study. Another assumption is the equal-environment assumption, that $\mathrm{MZ}$ and $\mathrm{DZ}$ twins are equally correlated for exposure to environmental factors of aetiological relevance for the trait under study. This may be reasonable to assume in infancy, childhood and adolescence, where the twins are reared in a common environment and exposed to potential risk factors for asthma (e.g. parental smoking and indoor allergens) to the same extent. In utero, monochorionic twins (exclusively MZ) may experience a more adverse intrauterine environment due to competition compared with dichorionic twins. However, in a study of 3,808 Australian twin pairs no difference in the prevalence of asthma or wheezing was found between single-placenta $\mathrm{MZ}$ twins and DZ twins or in concordance between single- and double-placenta MZ twins [24]. For adult twins the equal-environment assumption may be incorrect, as $\mathrm{MZ}$ twins spend more time together and may choose a similar occupation to a greater extent than DZ twins. This may cause an overestimate of the heritability if environmental risk factors for asthma are more often shared among MZ than among DZ twin pairs. There was no zygosity difference in the cumulative incidence of asthma in the present data, suggesting that there may be no marked effect of being an MZ twin compared to an DZ twin with regard to the environmental factors associated with an increased risk of asthma.

The aetiology of the disease under study must not differ between twins and singletons if the results from a twin study are to be applied to the general population. Concerns have been raised about the validity of the classic twin study according to the foetal origin hypothesis, which states that the risk of adult morbidity and mortality is heightened by retardation in intrauterine development. Associations between low birth weight and asthma [25] and low birth weight and adult forced expiratory volume in one second (FEV1) [26] support the hypothesis that suboptimal intrauterine conditions, which retard the foetal weight gain, may have long-term consequences on the growth and function of the airways. Twins are, on average, lighter than singletons at birth and may, therefore, have an increased risk for later respiratory disease such as asthma.
However, the cumulative incidence of asthma in the present study of twins corresponds to that found in population-based studies of Danish singletons. A cross-sectional study among 1,064 Danish schoolchildren aged 6-17 yrs [27] revealed a point prevalence of $4 \%$ of physician-diagnosed asthma. In addition, $3.2 \%$ of the children had bronchial hyperreactivity, indicating a point prevalence of asthma of $7.2 \%$, compared with a cumulative incidence of $7.0 \%$ in the present sample of twins, aged $12-17$ yrs. A population-based survey among 4,668 Danish individuals, aged $\geq 16$ yrs [28], showed a cumulative incidence of selfreported asthma of $7.0 \%$. Based on cumulative incidence, it was expected that this study would reveal an increase in asthma with age, which was not the case. The decreasing cumulative incidence with age for males and the lack of a trend across age for females may indicate an increase in the incidence of asthma over time, although it may be partly explained by an increased recall bias with age. Recall bias will tend to underestimate the heritability according to the above-mentioned effects of misclassification. The sex pattern, with a male dominance in childhood and adolescence changing to a female dominance among young adults, is widely reported among singletons. The consistency of estimates of cumulative incidence of asthma in the twin sample and the findings in studies of singletons suggests that the nature of low birth-weight in twins may be different from that in low birth-weight singletons with regard to the prediction of asthma. This supports the finding from other studies $[24,29]$ that the health of twins after the first year of life does not differ from singletons, emphasizing the validity of the classic twin method. Since no marked selection, information or comparison bias was demonstrated that could weaken the results of the present study, it seems reasonable to extend the results to the general population.

In conclusion, the lack of an increasing cumulative incidence of asthma with age in the present study may support a true increase in the prevalence of the disease. The results of the biometric modelling emphasize a major genetic influence on the liability to asthma; however a substantial proportion of the phenotypic variance is due to individualspecific environmental factors. Using the single question of ever having asthma, this study was not able to distinguish between the different asthmatic phenotypes which, despite central features such as airway inflammation, bronchial hyperresponsiveness and reversible airflow obstruction, may be manifestations of distinct diseases. This must be taken into account when evaluating the influence of genetic and environmental factors on the development of asthma. The various asthmatic phenotypes may be a result of gene-environment interactions. This refers to traits where the same set of genes is expressed differently under various environmental conditions or the same environment may have a greater effect on some genotypes than on others. The biometric modelling used in the present study is based on the assumption of no gene-environment interaction. If it were possible to stratify twins for being or not being exposed to a specific environmental factor, the importance of the gene-environment interaction for the development of asthma could be detected with structural equation modelling. However, this stratification would need large sample sizes, which could only be achieved in an international collaboration, to find significant evidence for gene-environment interactions. 
Acknowledgements. The authors would like to thank A. Green and H. Beck-Nielsen for overseeing the establishment of the Danish Twin Registry.

\section{References}

1. Nieminen MM, Kaprio J, Koskenvuo M. A populationbased study of bronchial asthma in adult twin pairs. Chest 1991; 100: 70-75.

2. Harris JR, Magnus P, Samuelsen SO, Tambs K. No evidence for effects of family environment on asthma. A retrospective study of Norwegian twins. Am J Respir Crit Care Med 1997; 156: 43-49.

3. Orlebeke JF, Koopmans JR, Boomsma DI, Erikson AW. Genetic analysis of asthma/bronchitis and allergy in a sample of adolescent twins: a study from the Dutch twin register (Abstract). Congress Proceedings, 7th International Congress on Twin Studies, Tokyo, Japanese Society for Twin Studies, 1992; p. 109.

4. Duffy DL, Martin NG, Battistutta D, Hopper JL, Mathews JD. Genetics of asthma and hay fever in Australian twins. Am Rev Respir Dis 1990; 142: 1351-1358.

5. Halken S, Høst A, Nilsson L, Taudorf E. Passive smoking as a risk factor for development of obstructive respiratory disease and allergic sensitization. Allergy 1995; 50: 97105.

6. Sporik R, Holgate ST, Platts-Mills TAE, Cogswell JJ. Exposure to house-dust mite allergen (der p1) and the development of asthma in childhood. N Engl J Med 1990; 323: 502-507.

7. Sears MR, Herbison GP, Holdaway MD, Hewitt CJ, Flannery EM, Silva PA. The relative risks of sensitivity to grass pollen, house dust mite and cat dander in the development of childhood asthma. Clin Exp Allergy 1989; 19: 419-424.

8. Ulrik CS, Backer V, Hesse B, Dirksen A. Risk factors for development of asthma in children and adolescence: findings from a longitudinal population study. Respir Med 1996; 90: 623-630.

9. Kyvik KO, Green A, Beck-Nielsen H. The new Danish Twin Register: establishment and analysis of twinning rates. Int J Epidemiol 1995; 24: 589-596.

10. Hauge M. The Danish twin register. In: Mednick S, Baert A, Bachmann B, eds. Prospective Longitudinal Research. An Empirical Basis for the Primary Prevention of Psychological Disorders. Oxford, Oxford University Press, 1981; pp. 217-221.

11. McGue M. When assessing twin concordance, use the probandwise not the pairwise rate. Schizophr Bull 1992; 18: 171-176.

12. Ramakrishnan V, Goldberg J, Henderson WG, et al. Elementary methods for the analysis of dichotomous outcomes in unselected samples of twins. Genet Epidemiol 1992; 9: 273-287.

13. Falconer DS. The inheritance of liability to certain dis- eases, estimated from the incidence among relatives. Ann Hum Genet 1965; 29: 51-76.

14. Neale MC, Cardon LR. Methodology for Genetic Studies of Twins and Families. Dordrecht, Kluwer Academic Publishers, 1992.

15. Neale MC. Mx: statistical modeling, 3rd Edn. Richmond, Medical College of Virginia, Virginia Commonwealth University, 1995.

16. Akaike H. Factor analysis and AIC. Psychometrika 1987; 52: 317-332.

17. Edfors Lubs ML. Allergy in 7000 twin pairs. Acta Allergol 1971; 26: 249-285.

18. Christian JC, Norton JA, Sorbel J, Williams CJ. Comparison of analysis of variance and maximum likelihood based path analysis of twin data: partitioning genetic and environmental sources of covariance. Genet Epidemiol 1995; 12: 27-35.

19. Lange P, Ulrik CS, Vestbo J. Mortality in adults with selfreported asthma. Copenhagen City Heart Study Group. Lancet 1996; 347: 1285-1289.

20. Huovinen E, Kaprio J, Vesterinen E, Koskenvuo M. Mortality of adults with asthma: a prospective cohort study. Thorax 1997; 52: 49-54.

21. Nystad W, Magnus P, Gulsvik A, Skarpaas IJ, Carlsen $\mathrm{KH}$. Changing prevalence of asthma in school children: evidence for diagnostic changes in asthma in two surveys 13 yrs apart. Eur Respir J 1997; 10: 1046-1051.

22. Manfreda J, Becker AB, Wang PZ, Roos LL, Anthonisen NR. Trends in physician-diagnosed asthma prevalence in Manitoba between 1980 and 1990. Chest 1993; 103: 151157.

23. Torén K, Brisman J, Järvholm B. Asthma and asthma-like symptoms in adults assessed by questionnaires. Chest 1993; 104: 600-608.

24. Duffy DL. Twin studies in medical research (Letter and Comment). Lancet 1993; 341: 1418-1419.

25. Schwartz J, Gold D, Dockery DW, Weiss ST, Speizer FE. Predictors of asthma and persistent wheeze in a national sample of children in the United States. Association with social class, perinatal events, and race. Am Rev Respir Dis 1990; 142: 555-562.

26. Barker DJ, Godfrey KM, Fall C, Osmond C, Winter PD, Shaheen SO. Relation of birth weight and childhood respiratory infection to adult lung function and death from chronic obstructive airways disease. BMJ 1991; 303: 671-675.

27. Høst AH, Duus T, Ibsen TB, Høst A. Asthma prevalence in school children. Is the disease underdiagnosed. Ugeskr Laeger 1993; 155: 3978-3981.

28. Keiding L. Asthma, Allergy, and Other Types of Hypersensitivity in Denmark - and the Development 19871994. Copenhagen, Danish Institute for Clinical Epidemiology, Copenhagen, 1997.

29. Christensen K, Vaupel JW, Holm NV, Yashin AI. Mortality among twins after age 6: fetal origins hypothesis versus twin method. BMJ 1995; 310: 432-436. 